Research Article

\title{
Maintenance Personnel Optimization Model of Vehicle Equipment Based on Support Task
}

\author{
Weixing Song $\mathbb{D}^{1,2}$ Zhengjun Lei, ${ }^{2}$ Qian Le, ${ }^{2}$ Fengyue $\mathrm{Li}^{3}{ }^{3}$ and Jingjing $\mathrm{Wu}^{4}$ \\ ${ }^{1}$ Army Engineering University of PLA, Shijiazhuang 050003, China \\ ${ }^{2} 32272$ Group of PLA, Lanzhou 730000, China \\ ${ }^{3}$ School of Mathematics and Statistics, Xidian University, Xi'an 710100, China \\ ${ }^{4}$ Western Theater Army Department of Logistics, Lanzhou 730000, China \\ Correspondence should be addressed to Weixing Song; 88159073@qq.com
}

Received 6 February 2021; Revised 25 March 2021; Accepted 4 May 2021; Published 13 May 2021

Academic Editor: Rohit Salgotra

Copyright $\odot 2021$ Weixing Song et al. This is an open access article distributed under the Creative Commons Attribution License, which permits unrestricted use, distribution, and reproduction in any medium, provided the original work is properly cited.

\begin{abstract}
Vehicle equipment maintenance support tasks have problems such as low maintenance efficiency and unreasonable allocation of maintenance personnel. In order to further strengthen the theoretical research of vehicle equipment maintenance support, an optimization model of vehicle equipment maintenance personnel based on support task is proposed in this paper. Firstly, the maintenance workload model of vehicle equipment is constructed by analyzing the three task sources of vehicle equipment: scheduled maintenance, natural random failure, and combat damage. Then, considering the technical professional level, maintenance efficiency, and other factors of maintenance personnel, two optimization models of maintenance personnel are constructed. In view of the situation where there are enough human resources, the prediction model of the number of personnel with the minimum total number as the goal is constructed to achieve the purpose of saving human resources. Using MATLAB mixed integer nonlinear programming problem (MINP) toolbox to solve the prediction model of the number of personnel, in view of the shortage of maintenance personnel, a maintenance personnel allocation model aiming at minimizing maintenance time is constructed to maximize maintenance efficiency. In order to solve the model, the fruit fly optimization algorithm (FOA) is improved, and the group cooperation is used to update the fruit fly position. The new algorithm not only retains the essential advantages of the FOA but also solves the problem that the algorithm is easy to fall into local extreme value and improves the global optimization ability of the algorithm. Finally, two example simulations verify the effectiveness of the optimization method in this paper and provide a certain theoretical basis for maintenance personnel to optimize decision-making.
\end{abstract}

\section{Introduction}

Entering the twenty-first century, information technology weapons and equipment have gradually developed, hightech weapon ratio has increased, and the corresponding equipment support forces have also been changed [1]. In order to improve the support capability of the maintenance support system and give full play to the effectiveness of the existing support resources, the allocation structure of the support force must be optimized and adjusted. The traditional maintenance support resources have a shortage of scientific calculation methods. They mainly rely on subjective experience, historical data comparison, and other methods and lack the support of quantitative methods, which leads to bad phenomena such as low maintenance efficiency and waste of resources [2].

As an important part of modern military weapons and equipment, vehicle equipment is one of the important factors to improve the combat effectiveness of the army. However, the current situation of vehicle equipment maintenance support is still difficult to meet the requirements of the new circumstances, and the corresponding theoretical research does not dive deep enough. There are still some problems in terms of vehicle equipment, such as low maintenance efficiency and unreasonable allocation of maintenance personnel. The optimization of maintenance 
personnel is an important research content of support force allocation. The reasonable allocation of maintenance personnel and the adjustment of the number of technical personnel at various levels can enable the maintenance organization to have a reasonable structure of technical personnel, support tasks can be completed in a timely and efficient manner, and equipment support can obtain maximum benefits. Maintaining and repairing the normal use function and technical condition of the vehicle is the main measure to ensure the combat capability of the vehicle equipment [3]. It is a realistic and meaningful work to do a good job in the optimization of vehicle equipment maintenance personnel.

The contents of the research on the optimization of maintenance support resources are varied, and the methods adopted are also different. It mainly includes three types: RCPSP (Resource-Constrained Project Scheduling Problem), inventory control of maintenance spare parts, and prediction optimization of maintenance personnel.

The RCPSP is a scheduling optimization problem in an environment where maintenance resources have been determined. In the aspect of modeling and solving the RCPSP problem, researchers mainly establish the multiconstrained programming model and use the heuristic algorithm to study. References [4,5] study the MRCPSP (Multimode Resource-constrained Project Scheduling Problem), considering human, machinery, equipment, and consumptive resources, and use the meta-heuristic algorithm to solve these problems effectively. The PRCPSP (Preemptive Resource-Constrained Project Scheduling Problem) mathematical model aiming at the shortest maintenance time and the minimum total load of maintenance personnel is established in references [6-8]. Literature [9] introduces GPRs (Generalized priority relation) and AON (ActivityOn-Node) to describe the sequential relationship of tasks and uses an improved cuckoo algorithm to solve the problem. Literature [10] improves the tabu search algorithm and solves the RCPSP model. Literature [11] considers the resource constraints such as personnel, support equipment, workstation space, and replenishment resources and establishes a mathematical model of RSPFDO (robust scheduling problem for flightdeck operation), with the goal of maximizing the probability of completion and minimizing the expected completion time.

In terms of inventory control decision-making process of maintenance spare parts optimization, there are mainly two kinds of researches, one is METRIC (Multiechelon Technique Recoverable Item Control) series model and the other is the series model of EOQ (Economic Order Quantity) theory. Papers [12, 13] propose a METRIC inventory model based on task analysis for the inventory allocation problem of repairable spare parts for aircraft equipment and solve the model using the marginal analysis method. Literature [14] considers the dynamic changing process of spare parts requirements during combat missions and establishes a task-oriented time-varying availability model of spare parts. Literature [15] establishes an inventory optimization model of military aircraft maintenance spare parts under source constraints but did not consider the repairability of spare parts. Papers [16, 17] establish a joint maintenance and spare parts inventory optimization model to fill this gap.

Research on the optimal allocation of maintenance personnel is of great significance for improving system maintenance efficiency and exerting resource efficiency. However, the existing personnel optimization research focuses on the enterprise or industrial background [18] and does not consider the maintenance task of the equipment. The established model is mainly aimed at nonmaintainable professional technical personnel, which is not suitable for the optimization of maintenance personnel of military equipment [19]. The optimized configuration of maintenance personnel can enable the maintenance organization with a reasonable structure of technical personnel and effective maintenance efficiency. In the aspect of optimization for the maintenance personnel resource configuration, the commonly used methods include maintenance task analysis method, queuing theory method, maintenance unit method, and various mathematical programming models. According to the division of personnel specialties in $[20,21]$, a personnel quantity prediction model based on the minimum maintenance unit method is established. Papers [22, 23] analyze the maintenance support process and construct a maintenance personnel allocation model and a demand forecast model based on queuing theory. Literature [11] analyzes the influencing factors and restrictive factors of ship equipment, establishes a maintenance personnel optimization model with the smallest cumulative repair time, and solves the model by using a hybrid genetic algorithm. Literature [24] proposes an optimization model for maintenance personnel to minimize labor costs and designs an enhanced mixed integer linear programming algorithm to solve the model. The research on the optimal configuration of maintenance personnel is usually from the analysis of equipment maintenance tasks [25-27]. Accurate analysis of equipment maintenance support tasks is the prerequisite for formulating equipment support programs.

At present, the problem of optimal allocation of resources has been applied in many fields, among which maintenance support personnel is the main force and core of equipment maintenance work [28]. In the actual vehicle equipment maintenance activities, the general practice is to assign maintenance tasks to fixed maintenance personnel, resulting in problems such as dispersion of maintenance activities and overtime of task completion. However, by increasing the number of personnel to improve the maintenance speed, it is easy to lead to the waste of human resources. The maintenance personnel optimization problem can be discussed in two situations. In the case of sufficient human resources, how to avoid the waste of human resources is the focus of our research, and in the case of human resource shortage, the study of maximizing maintenance efficiency becomes more meaningful. Therefore, this paper establishes an optimization model of vehicle equipment maintenance personnel for the above two situations. 
The main contributions of this research are as follows:

(1) The maintenance task source of vehicle equipment and the determination process of maintenance personnel optimization are analyzed in detail, and the maintenance workload model of vehicle equipment is constructed, which mainly includes scheduled maintenance, natural random failure, and combat damage.

(2) In order to avoid the waste of human resources, in view of the maintenance tasks with enough human resources and aiming at minimizing the total number of maintenance personnel, a prediction model for the number of maintenance personnel is constructed. The model can be used to solve the number of maintenance personnel at each professional level, minimize the number of maintenance personnel, and meet the cumulative maintenance time limit.

(3) In order to maximize maintenance efficiency, in view of the shortage of maintenance human resources and the diversity of professional skills, an optimal allocation model of maintenance personnel with the goal of minimizing the cumulative repair time is constructed. The model can be used to allocate limited maintenance personnel to maximize maintenance efficiency and meet maintenance time constraints.

(4) The traditional fruit fly optimization algorithm (FOA) is improved by using the location update method of group collaboration, which improves the defect as the algorithm is easy to fall into local extreme value. The simulation results show that the improved FOA has good solution efficiency, and the personnel allocation scheme can effectively shorten the equipment maintenance time.

\section{Analysis of Maintenance Tasks of Vehicle Equipment}

Scientific analysis of vehicle equipment maintenance tasks is the basis of human resources optimization. The determination process of personnel resources in maintenance support is shown in Figure 1. First, group the damage types of vehicle equipment, and estimate the workload of each group of maintenance tasks based on information such as the use plan of the vehicle equipment during the guarantee period, the initial state, the maintenance standard, and the failure rate of the scheduled maintenance. Then, according to the existing maintenance personnel and actual needs, the number of maintenance personnel is predicted and so is the configuration of staffing.

The use phase of vehicles includes the daily training phase and combat phase, mainly for preventive maintenance and damage repair. Due to the relatively long period of time in the daily training phase, the sources of maintenance tasks mainly include scheduled preventive maintenance and natural fault repair. In addition, the source of maintenance tasks in the combat phase mainly includes combat damage repairs.
Scheduled repairs include minor repair, medium repair, and overhaul, which are determined by the intensity of vehicle use. The intensity of use is related to the working hours of the vehicle. Minor repair refers to the activity of maintaining the tactical and technical performance of equipment; medium repair refers to the activity of restoring the tactical and technical performance of equipment; overhaul refers to the activity of fully restoring the tactical and technical performance of equipment [29]. Taking a certain type of armored vehicle as an example, the ratio of the number of major, medium, and minor repairs during each major repair interval is $1: 1: 4$, as shown in Figure 2 . After general overhaul, the vehicle is equipped as new and the working hours are reset to zero.

Natural failure and combat damage maintenance are restorative maintenance, which refers to damage failures that have not been used until the specified repair interval. Natural failures are often closely related to the working hours of vehicles. The law of vehicle fault distribution includes Poisson distribution, normal distribution, and Weibull distribution. The failure rate based on vehicleworking hours can be predicted by vehicle historical fault data and some existing prediction models, such as neural network, ARMA model, and grey prediction model [30, 31]. Generally speaking, the longer the vehicle equipment engine works, the greater the probability of natural random failure.

Combat damage repair is mainly to repair vehicles damaged by weapons in the combat phase. According to the level of damage, it can be divided into complete damage, severe damage, moderate damage, and minor damage. In general, the relationship between the damage rate of vehicle equipment and combat intensity is shown in Figure 3.

\section{Vehicle Maintenance Workload Model}

Scientifically and reliably predicting the maintenance workload of vehicle equipment is the basis and prerequisite for formulating an appropriate maintenance personnel plan [32]. It is assumed that the damage attributes of vehicle equipment during the daily training phase and the combat phase include six types of damage types: chassis, ordnance, fire control, communications, photoelectric, and electrical appliances. The subscript $k$ represents the vehicle equipment index. The letter $j$ is used for the index of scheduled maintenance range; $j=1,2,3$, respectively, indicates minor repair, medium repair, and overhaul. Use $s$ to represent the index of combat damage range; $s=1,2,3$, respectively, represent the three ranges of light damage, moderate damage, and severe damage. The subscripts $i, f$, and $g$ are, respectively, the index of scheduled maintenance failure type, natural random failure type, and combat damage failure type.

3.1. Scheduled Maintenance Workload. Scheduled maintenance workload includes minor repair, medium repair, and overhaul tasks, which are mainly calculated according to the use plan and maintenance plan. Assuming that the number of $j$-range maintenance personnel generated by $Q$ vehicles of 

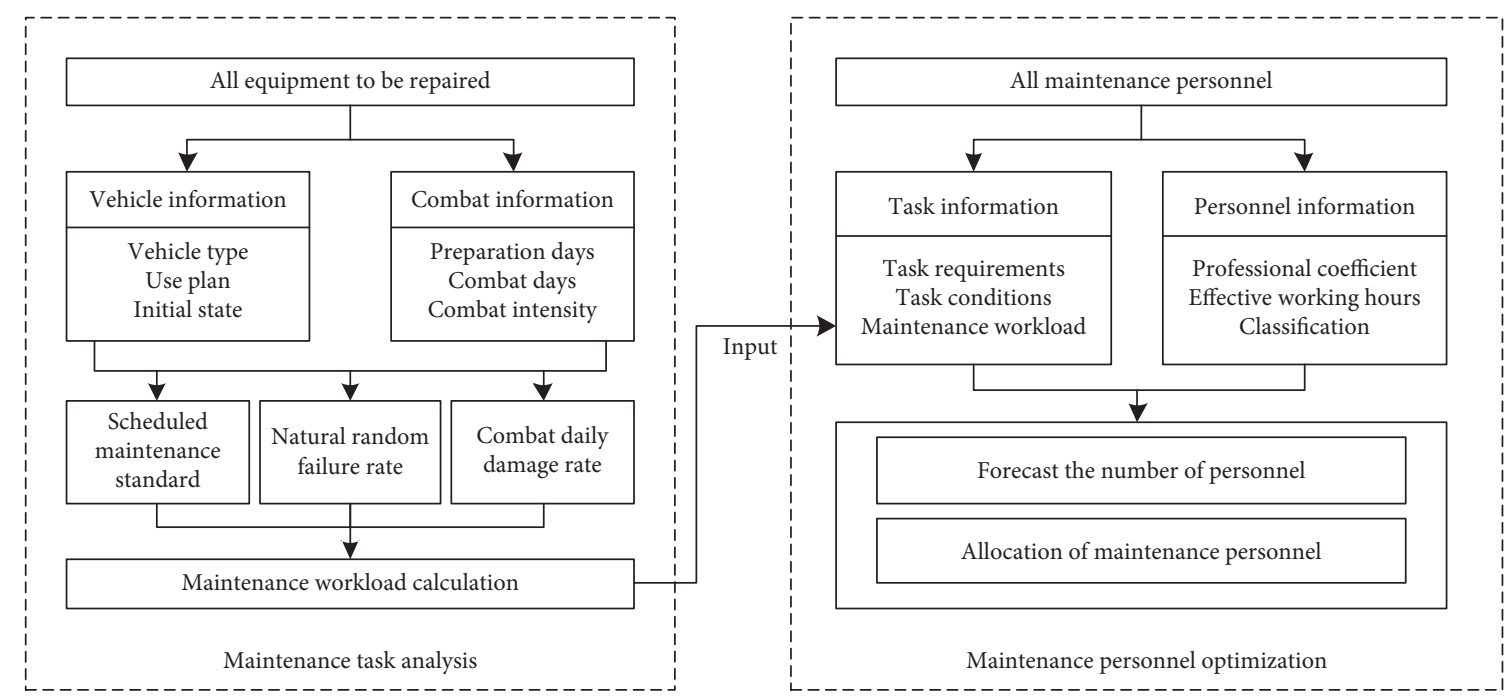

FIGURE 1: The determination process of personnel resources in maintenance support.

the same type during the guarantee time is $Z_{j}$, the calculation method for $Z_{j}$ is as follows:

$$
Z_{j}=\sum_{k=1}^{Q}\left\lfloor\frac{M_{k}-M_{j k}+\mathrm{EM}_{k}}{(1+\sigma) \mathrm{IM}_{j}}\right\rfloor
$$

where $M_{k}$ represents the current use time of the $k$-th vehicle equipment. $M_{j k}$ represents the engine working time of the last $j$-range maintenance or higher than $j$-range maintenance of $k$-th vehicle equipment. $\mathrm{EM}_{k}$ represents the estimated time of use of the $k$-th vehicle equipment during the guarantee period; $\mathrm{IM}_{j}$ represents the maintenance interval of range $j ; \sigma$ is the correction factor of the maintenance interval, which can be taken as 0 in the daily training phase and $30 \%$ to $50 \%$ in the combat phase. Therefore, the calculation method of each type of failure maintenance number $S_{j i}$ and maintenance workload $\mathrm{Cl}_{j i}$ in the scheduled maintenance is as follows:

$$
\begin{aligned}
S_{j i} & =Z_{j}{ }^{*} \gamma_{j i}, \\
C l_{j i} & =t_{j i}{ }^{*} S_{j i} .
\end{aligned}
$$

In the formula, $\gamma_{j i}$ is the proportion of maintenance times of damage type $i$ in $j$-range scheduled maintenance and $t_{j i}$ is the rated man-hour of damage type $i$ in $j$-range scheduled maintenance.

3.2. Natural Random Failure Maintenance Workload. The random failure rate of vehicle equipment has a direct relationship with factors such as vehicle failure rules, equipment technical status, and use conditions, and it mainly depends on the intensity of vehicle equipment use. This paper uses the working time of the engine to measure the intensity of vehicle usage. Divide a major repair interval $\mathrm{IM}_{3}$ into segments every 100 hours. $l$ is the index of the segmented interval, $l=1,2, \ldots,\left\lfloor\mathrm{IM}_{3} / 100\right\rfloor+1 . \lambda_{i}$ represents the failure rate in the $l$-th time period. $f$ is the index of the

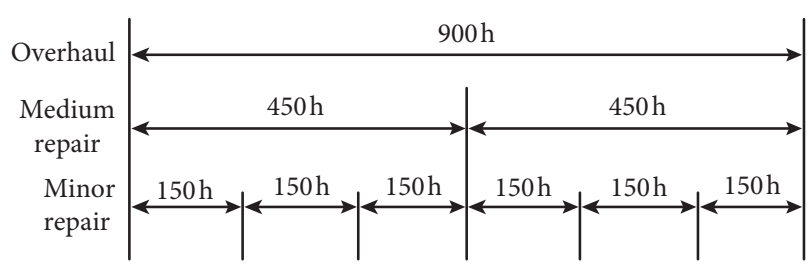

FIgURE 2: Schematic diagram of maintenance interval.

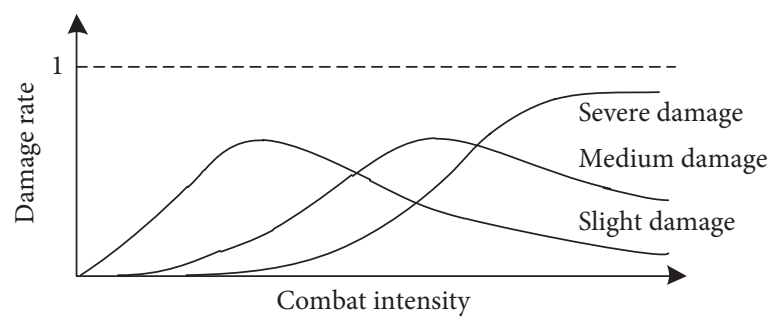

FIGURE 3: Relationship between equipment damage rate and combat intensity.

fault type, and the fault proportion is $\beta_{f}$. The natural failure maintenance workload is determined by the following method.

Therefore, the natural random failure maintenance number $H_{f}$ can be determined by the following formula:

$$
H_{f}=\sum_{\forall l} \sum_{\forall k} \Delta M_{k l}^{*} \lambda_{l}^{*} \beta_{f},
$$

where $\Delta M_{k l}$ is the use time of the $k$-th vehicle equipment in the $k$-th segmented interval. The calculation method is as follows (Algorithm 1).

Therefore, the maintenance workload for natural random failures $\mathrm{Cn}_{f}$ is as follows:

$$
C n_{f}=H_{f} \cdot c_{f},
$$

where $c_{f}$ is the rated man-hour of vehicle fault $f$. 


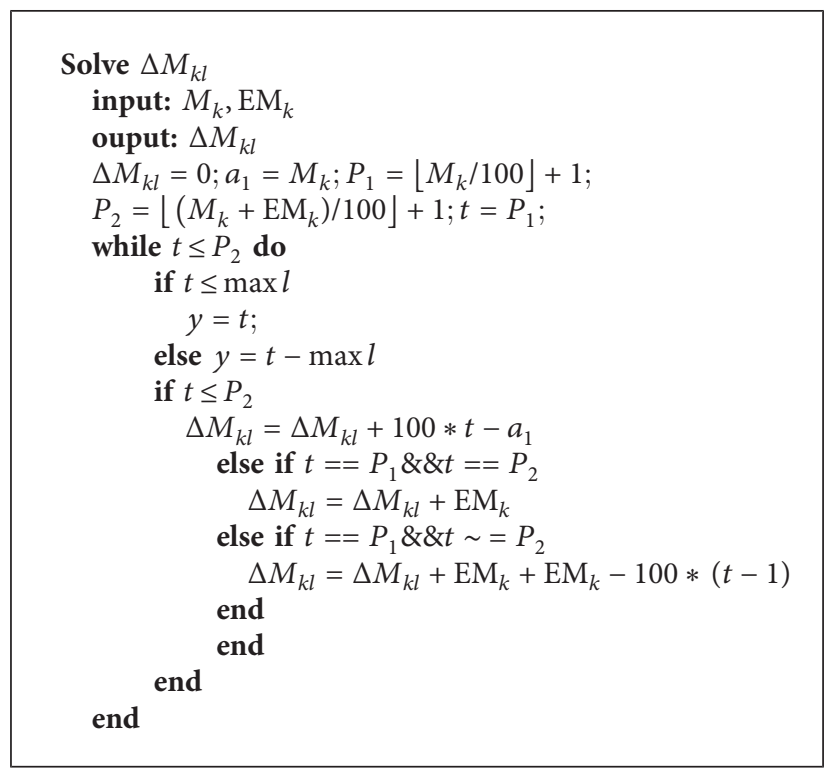

Algorithm 1

3.3. Combat Damage Maintenance Workload. During combat time, due to the attack of enemy weapons and equipment, the vehicle equipment is damaged. Vehicle combat damage is affected and controlled by many factors such as the task of the army, the technical status of the vehicle, the quality of the operators, the comparison of weapons and forces, and the operating conditions of the vehicle. Due to the numerous influencing factors and the dynamic changes of the battlefield environment, it is difficult to accurately calculate the amount of vehicle combat damage. The general practice of various countries on combat damage estimation is usually to calculate probability according to a given combat damage rate. The calculation methods for the number of combat damage repairs $N_{g}$ and the combat damage repair workload $C f_{g}$ are as follows:

$$
\begin{aligned}
N_{s g} & =Q^{*} T_{0}{ }^{*} \lambda^{*} \rho_{s}{ }^{*} \rho_{s g}, \\
C f_{s g} & =N_{s g}^{*} r_{s g},
\end{aligned}
$$

where $T_{0}$ indicates the duration of the battle. $\lambda$ is the daily combat damage rate. $\rho_{j}$ is the proportion of $j$-range repairs in war-damaged equipment. $\rho_{s}$ is the $s$-range combat damage rate. $\rho_{s g}$ is the ratio of the $g$-th damage type in $s$ range combat damage, and $r_{s g}$ is the rated man-hours for the $g$-th failure repair in $s$-range combat damage.

3.4. Calculation of Maintenance Workload in Each Group. The maintenance tasks are grouped according to the type of failure. For the convenience of presentation, the subscript $z$ is used to indicate the grouping index $z=1,2, \ldots, 6$, respectively, represent the maintenance group of six types of failures of chassis, ordnance, fire control, communication, photoelectric, and electrical appliances. $Y_{s}$ represents $z$-class failure set. The maintenance workload of $z$-group $C_{z}$ is calculated as follows:

$$
C_{z}=\sum_{\forall i \in Y_{z}} \sum_{j=1}^{3} C l_{j i}+\sum_{\forall f \in Y_{z}} C n_{f}+\sum_{\forall g \in Y_{z}} \sum_{s=1}^{4} C f_{s g} .
$$

\section{Optimization Model of Maintenance Personnel for Vehicle Equipment}

In the maintenance tasks with sufficient human resources, there will often be a realistic problem of wasting human resources caused by arranging too many personnel to work. In this case, it would be more meaningful to meet the maintenance time constraints by arranging the number of maintenance personnel in each group reasonably. The maintenance tasks with scarce human resources: it is often necessary to involve as many personnel as possible in the maintenance tasks. In this case, it would be more meaningful to reduce maintenance time by reasonably assigning maintenance personnel to each group.

4.1. Forecast Model of the Number of Maintenance Personnel. The knowledge and experience of maintenance personnel play an important role in the efficiency and safety of maintenance support tasks [33]. The maintenance personnel are each profession is divided into different grades, and $r=$ $1,2,3$ are used to represent junior, intermediate, and senior, respectively. The professionalism of the maintenance personnel is consistent with the six types of failures, and the subscript $z$ is used to indicate the professionalism of the maintenance personnel. It is known that the average specialization level is coefficient $\theta_{z r}$ and the average daily working time is $v_{r}$ of $z$-profession-r-grade maintenance 
personnel. Then the repair efficiency $V_{z r}$ of $z$-profession- $r$ grade maintenance personnel is calculated as follows:

$$
V_{z r}=\theta_{z r}^{*} v_{z r}
$$

Matrix $\mathbf{X}$ is used to express the number distribution scheme of maintenance personnel of each profession and grade, $X_{z}$ represents the number distribution scheme of personnel at all grades in $z$-group, and $x_{z r}$ represents the number of $r$-grade maintenance personnel in $z$-group.

$$
\mathbf{X}=\left[\begin{array}{c}
X_{1} \\
\vdots \\
X_{z} \\
\vdots \\
X_{6}
\end{array}\right]=\left[\begin{array}{ccc}
x_{11} & x_{12} & x_{13} \\
\vdots & \vdots & \vdots \\
x_{z 1} & x_{z 2} & x_{z 3} \\
\vdots & \vdots & \vdots \\
x_{61} & x_{62} & x_{63}
\end{array}\right]
$$

Then, under the $\mathbf{X}$ personnel allocation scheme, the cumulative repair time in $z$-group is as follows:

$$
T_{z}^{\prime}(\mathbf{X})=\frac{C_{z}}{\sum_{r=1}^{3} x_{z r} V_{z r}} .
$$

Due to the independence of vehicle equipment damage assessment, the increase in the number of maintenance personnel does not have obvious benefits in shortening the assessment time. Therefore, when multiple maintenance personnel evaluate a vehicle at the same time, the minimum evaluation time of the personnel is usually taken as the evaluation time of the group $T_{z}^{\prime \prime}$.

$$
T_{z}^{\prime \prime}(\mathbf{X})=\mathrm{NL}_{z}^{*} \min _{\forall x_{z r}} t_{z r}(\mathbf{X})
$$

where $t_{z r}$ represents the average time for each level of maintenance personnel to assess the damage in $z$-group. $\mathrm{NL}_{z}$ is the amount of damages in group $Z$. Therefore, the total repair time of each group is as follows:

$$
T_{z}(\mathbf{X})=T_{z}^{\prime}(\mathbf{X})+T_{z}^{\prime \prime}(\mathbf{X})
$$

In summary, a prediction model of maintenance personnel quantity is established with the minimum number of total personnel as the objective function, and the number of existing maintenance personnel, cumulative repair time, and professional restrictions as constraints.

$$
\begin{aligned}
& \min \sum_{z} \sum_{j} x_{z j}, \\
& \text { s.t. } \\
& \left\{\begin{array}{l}
x_{z r} \leq a_{z r}, \quad z=1,2, \ldots, 6, r=1,2,3, \\
\sum_{z=1}^{6} T_{z}(\mathbf{X}) \leq t_{0}, \\
T_{z}(\mathbf{X}) \leq \omega_{z}^{*} t_{0}, \quad z=1,2, \ldots, 6, \\
x_{z j} \in N, \quad z=1,2, \ldots, 6, j=1,2,3 .
\end{array}\right.
\end{aligned}
$$

In the formula, constraint condition 1 limits the number of personnel; condition 2 is the total cumulative repair time limit; conditions 3 is the cumulative repair time limit of $z$ - group, here requires $\omega_{z} \in(0,1)$; condition 4 is the basic attribute restriction of decision variables.

4.2. Maintenance Personnel Allocation Model. In actual maintenance support activities, there is a shortage of maintenance personnel and their professionalism is not unique. Under such circumstances, it is more meaningful to study how to allocate limited maintenance personnel to maximize maintenance efficiency. It is assumed that the professional level coefficient of $N$ maintenance personnel for each type of damage, the effective working hours per day, the evaluation time, and the cooperation coefficient is known.

Generally speaking, due to the differences in knowledge, experience, and tacit understanding of different maintenance personnel, the cooperative maintenance efficiency is usually less than the sum of the efficiency of various maintenance personnel. The subscript $p$ represents the index of maintenance personnel. The value is related to the cooperation coefficient between maintenance personnel, so the cooperative maintenance efficiency $V_{z}^{\prime}$ in $z$-group of $x$ maintenance personnel can be obtained.

$$
V_{z}^{\prime}=\sum_{p=1}^{x} \theta_{z p}{ }^{*} v_{p}^{*} \xi
$$

$\theta_{z p}$ represents the coefficient of professionalization level of the $p$ th maintenance personnel for $z$-group failures; $v_{p}$ is the average daily working time of the $p$ th maintenance personnel; the cooperation coefficient $\xi$ takes the average of the cooperation coefficient of $x$ personnel.

The personnel allocation scheme is represented by a vector $\bar{x}$.

$$
\bar{x}=\left[x_{1}, x_{2}, \ldots, x_{p}, \ldots, x_{N}\right]
$$

$x_{j}$ indicates that the $j$-th maintenance personnel is assigned to work in $x_{j}$-group, $x_{j} \in\{1,2, \ldots, 6\}$. Then, under the personnel allocation scheme $\bar{x}$, the cumulative repair time of $z$-group is $T 1_{z}$.

$$
T 1_{z}(\bar{x})=\frac{C_{z}}{V_{z}^{\prime}} .
$$

Similar to the previous one, the evaluation time of each group $T 2_{z}$ can be solved.

$$
T 2_{z}(\bar{x})=\min _{\forall x_{e} \in z} t_{z}^{\prime}(\bar{x})^{*} \mathrm{NL}_{z} .
$$

In summary, a maintenance personnel allocation model with the minimum cumulative repair time as the objective function and the constraints on the cumulative repair time of each group is established as follows:

$$
\begin{aligned}
& \min T(\bar{x})=\sum_{z=1}^{6} T 1_{z}(\bar{x})+\sum_{z=2}^{6} T 2_{z}(\bar{x}), \\
& \text { s.t. } \\
& T 1_{z}(\bar{x})+T 2_{z}(\bar{x})<T(\bar{x})^{*} \varpi_{z}, \quad z=1,2, \ldots, 6 .
\end{aligned}
$$

The constraint condition indicates that the cumulative repair time of group $z$ shall not exceed $\omega_{z}$ times of the total repair time. 


\section{Algorithm and Case Analysis}

5.1. Algorithm Design. The relationship between the decision variables of the maintenance personnel quantity prediction model in Section 4.1 is relatively simple, so it is directly solved by MATLAB's mixed integer nonlinear optimization (MINP) toolbox OPTI. The decision-making variable relationship of the security staffing model in Section 4.2 is more complicated, and here we choose an improved FOA to solve the problem.

Swarm intelligence (SI) is an important part of computational intelligence, which is mainly used to solve optimization problems and has remarkable performance in highly complex problems $[34,35]$. Compared with other SI algorithms, the optimization mechanism of the FOA is simple and easy to understand, and the program code is easy to implement and needs to adjust fewer parameters [36]. However, the common problem of all unimproved intelligent algorithms is that they are prone to premature convergence or slow optimization. It is found that multipopulation coevolution and adaptive evolution strategies are effective ways to improve the search efficiency of intelligent algorithms, in which coevolution strategy plays a historic role in the field of biological heuristics [11]. In order to solve this defect of the fruit fly algorithm, the fruit fly algorithm is improved. Three flight directions are introduced, and the algorithm solving efficiency is improved through group collaboration. Figure 4 shows the basic flowchart of IFOA. Specific steps are as follows:

Step 1. Initialize the parameters.

Set the population size, the maximum number of iterations, the range of personnel allocation IR, and the judgment value of the flight directions $M_{1}, M_{2}$. Set the position vector $X_{i}=\left(x_{i}^{1}, x_{i}^{2}, \ldots, x_{i}^{N}\right)$ of each fruit fly individual $i$, which corresponds to the distribution plan of $N$ maintenance personnel, as shown in Figure 5.

$$
X_{-} \text {axis }=\operatorname{rand}(\mathrm{IR}) \text {. }
$$

Step 2: Use group collaboration to update the position of fruit flies.

Due to the complexity of the individual position vector of fruit fly, the convergence speed is slow and it is easy to fall into the local extreme value. In order to improve the search efficiency of the algorithm, according to the characteristics of the actual problem, three kinds of flight directions are introduced to carry out the position update operation, so as to realize the collaboration of the fruit fly group.

$$
X_{i}= \begin{cases}X_{i}+\operatorname{rand}\left(\mathrm{FR}_{1}\right), & \operatorname{sort}\left(X_{i}\right) \geq M_{1}, \\ X_{i}+\operatorname{rand}\left(\mathrm{FR}_{2}\right), & M_{1} \geq \operatorname{sort}\left(X_{i}\right) \geq M_{2}, \\ X_{-} \text {axis }+\operatorname{rand}\left(\mathrm{FR}_{3}\right), & \text { otherwise, }\end{cases}
$$

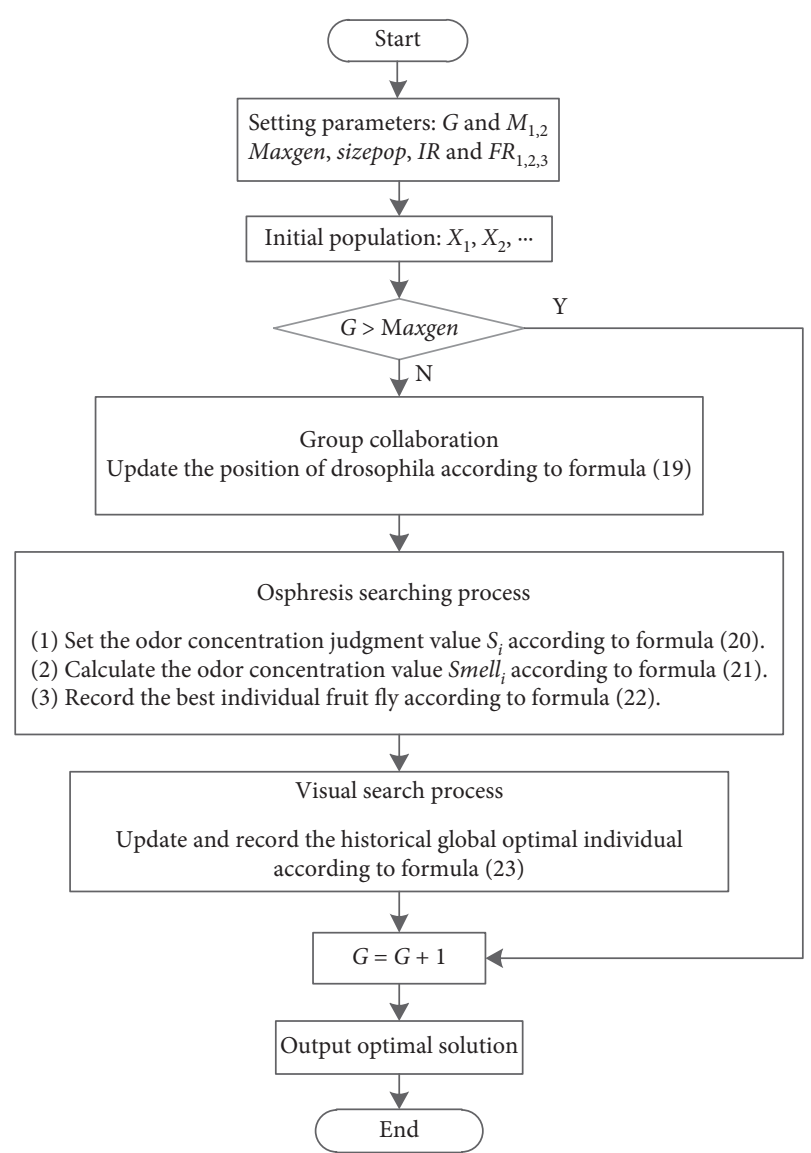

Figure 4: IFOA basic flow chart.

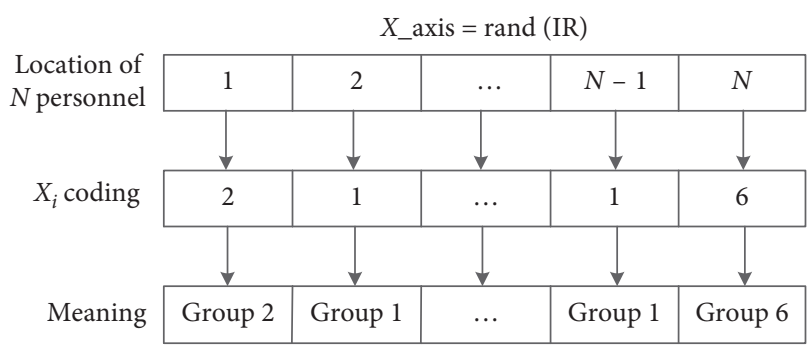

FIgURE 5: Schematic diagram of the coding scheme.

where sort $\left(X_{i}\right)$ refers to the order of the taste concentration values of individual fruit flies in the population. The first flight direction, $\mathrm{FR}_{1}$, is determined by the range $I R$, which can maintain the diversity of the population and improve the global search capability. The second flight direction, $\mathrm{FR}_{2}$, is determined by the maintenance workload of each group. The more the maintenance workload is, the easier it is to assign maintenance personnel. While maintaining the diversity of the population, it is easier to find the best location area. The third flight direction, $\mathrm{FR}_{3}$, is 
determined by the historically optimal fruit fly individuals. Individuals with better taste concentration values fly to the best position to maintain the optimality of the population and accelerate the algorithm convergence.

Step 3: Osphresis searching process.

Step 3.1: Calculate the judgment value of odor concentration $S_{i}$.

The actual problem needs to allocate a large number of personnel, and the location vector of individual fruit fly will become complex. In order to make the calculation simple and effective and to solve the defect of uneven distribution of odor concentration judgment value $S_{i}$ in the solution space, this paper gives up the calculation of fruit fly distance $D_{i}$ and directly assigns the fruit fly position vector $X_{i}$ to $S_{i}$.

$$
S_{i}=X_{i} \text {. }
$$

Step 3.2: Calculate the odor concentration of the fruit fly.

The objective function value of the individual fruit fly is as small as possible. The objective function of the personnel allocation model can be set as the odor concentration determination function of the algorithm. For the individual $X_{i}$ that does not meet the constraints, the concentration can be made equal to the maximum possible value of the objective function.

$$
\text { Smell }_{i}= \begin{cases}T\left(S_{i}\right), & S_{i} \text { satisfies the constraint, } \\ \max T, & \text { Otherwise. }\end{cases}
$$

Step 3.3: Record the best individual fruit fly.

$$
[\text { Bestsmell, Bestindex }]=\min \left(\text { Smell }_{i}\right)
$$

Step 4: Visual search process.

Compare the current optimal value Bestsmell to update and record the historical global optimal value Smellbest and individual location $X_{-}$axis.

$$
\begin{aligned}
\text { if Bestsmell } & <\text { Smellbest } \\
\text { Smellbest } & =\text { Bestsmell } ; \\
X_{-} \text {axis } & =X(\text { Bestindex }) ; \\
\text { end. } &
\end{aligned}
$$

Step 5: Repeat steps 2-4 until the maximum number of iterations, that is, $M>$ Maxgen, stop iteration.

5.2. Case Simulation. In order to verify the superiority of the models in this paper, two cases are generated based on the simulation data of the vehicle equipment of an army. Set the daily training time to 60 days and the combat time to 5 days. It requires 10 training vehicles and 5 combat readiness vehicles. The training vehicles are only used in the daily training phase and the combat readiness vehicles are only used for the combat phase. In addition, only slight combat damage is considered in this experiment. The basic data of vehicle parameters are shown in Table 1. Part of the data comes from literature [11].
Table 1 randomly generates the vehicle equipment parameters of this case simulation and uses the model in this paper to calculate the workload of this vehicle equipment maintenance task. The results are shown in Table 2. The maintenance workload for the six groups of damage types, chassis, ordnance, fire control, communications, photoelectric, and electrical, are $1240 \mathrm{~h}, 1450 \mathrm{~h}, 1117 \mathrm{~h}, 1065 \mathrm{~h}$, $1955 \mathrm{~h}$, and $1660 \mathrm{~h}$, respectively.

5.2.1. Case Simulation 1. Assuming that there are sufficient personnel who can participate in the maintenance activities this time, in order to save human resources, it is necessary to predict the minimum total number of maintenance personnel and the number of junior, intermediate, and senior personnel in each professional for this vehicle equipment maintenance support task. The total cumulative repair time must not exceed 97 days, and $\omega_{1}=0.13, \omega_{2}=0.16$, $\omega_{3}=0.13, \omega_{4}=0.11, \omega_{5}=0.30$, and $\omega_{6}=0.25$. Set personnel parameters according to expert experience. The daily working time is 12 hours and the evaluation time range is $U[0.5,3]$. Other personnel parameter settings are shown in Table 3.

Use the model in Section 4.1 to predict the number of maintenance personnel configuration, and use MATLAB's mixed integer nonlinear programming toolbox OPTI to solve the number of personnel prediction model. After 9534 iterations and 648 nodes, the best target value is 60 . The solution is shown in Table 4.

It can be seen from the solution result that the target value is 60 people. That is, it is arranged that the junior, intermediate, and senior maintenance personnel of the chassis specialty are 8,1 , and 2 , respectively, the junior, intermediate, and senior maintenance personnel of the ordnance specialty are 6,2, and 3, respectively; the junior, intermediate, and senior maintenance personnel of the fire control specialty are 4, 4, and 2, respectively; the junior, intermediate, and senior maintenance personnel of the communication specialty are 6,4 , and 2, respectively; the junior, intermediate, and senior maintenance personnel of the photoelectric specialty are 2, 4, and 1, respectively; and the junior, intermediate, and senior maintenance personnel of the electrical specialty are 5, 2, and 2, respectively. This configuration scheme can meet the needs of maintenance tasks. In order to verify the global optimal solution, the Lingo 7.0 software is used to solve the model again, and the result is consistent with Table 4 .

5.2.2. Case Simulation 2. Assuming that the number of personnel who can participate in the maintenance activities is limited, in order to maximize the maintenance efficiency, staffing needs to be optimized to minimize the total cumulative maintenance time and $\omega_{1}=0.3, \omega_{2}=0.4, \omega_{3}=0.3$, $\omega_{4}=0.3, \omega_{5}=0.5$, and $\omega_{6}=0.4$. Set personnel parameters according to expert experience. The number of security personnel is limited to 40 , the daily working time is 12 hours, and the evaluation time range is $U[0.5,3]$. The professional coefficient of 40 people is shown in Table 5. Use the model in Section 4.2 to optimize the maintenance personnel 
TABLE 1: Vehicle equipment parameters.

\begin{tabular}{lc}
\hline Vehicle equipment information & Value range \\
\hline Engine initial use time $M_{k}$ & $U[100,800]$ \\
Estimated use time during daily training $\mathrm{EM}_{k}$ & $U[100,400]$ \\
Scheduled maintenance interval $\mathrm{IM}_{j}$ & $250,500,1000$ \\
Minor repair failure type ratio $\gamma_{1 i}$ & $5: 2: 2: 1: 1: 1$ \\
Medium repair failure type ratio $\gamma_{2 i}$ & $10: 5: 3: 3: 2: 2$ \\
Overhaul failure type ratio $\gamma_{3 i}$ & $5: 5: 2: 2: 5: 2$ \\
Segment natural failure rate $\lambda_{l}$ & $U[0.0005,0.005]$ \\
Natural failure type ratio $\beta_{f}$ & $4: 5: 3: 4: 1: 1$ \\
Combat damage rate $\rho_{1}$ & 0.2 \\
Mild combat failure type ratio $\rho_{1 g}$ & $4: 3: 3: 3: 3: 8$ \\
Rated maintenance man-hours $t_{1 i} ; t_{2 i} ; t_{3 i} ; c_{f} ; r_{1 q}$ & $U[40,85], U[60,150], U[10,70], U[40,85]$, and $U[70,200]$ \\
\hline
\end{tabular}

TABLE 2: Maintenance workload of each group.

\begin{tabular}{lcccccc}
\hline Group & 1 & 2 & 3 & 4 & 5 & 6 \\
\hline Maintenance workload $(\mathrm{h})$ & 1240 & 1450 & 1117 & 1065 & 1955 & 1660 \\
\hline
\end{tabular}

TABLE 3: Case 1 personnel parameters.

\begin{tabular}{lccc}
\hline & Junior personnel & Intermediate personnel & Senior personnel \\
\hline & \multicolumn{2}{c}{ Average specialization coefficient } & 0.81 \\
Chassis & 0.79 & 0.80 & 0.95 \\
Ordnance & 0.74 & 0.81 & 0.93 \\
Fire control & 0.66 & 0.80 & 0.94 \\
Communication & 0.64 & 0.89 & 0.93 \\
Photoelectric & 0.79 & 0.81 & 0.96 \\
Electrical & 0.71 & Number of personnel & \\
\hline & & & 0.91 \\
Chassis & 90 & 3 & 2 \\
Ordnance & 52 & 4 & 2 \\
Fire control & 91 & 4 & 2 \\
Communication & 35 & 4 & 2 \\
Photoelectric & 90 & 2 & 2 \\
Electrical & 76 & & 2 \\
\hline
\end{tabular}

TABle 4: Personnel number model solution results.

\begin{tabular}{lccc}
\hline & Junior personnel & Intermediate personnel & Senior personnel \\
\hline Chassis & 8 & 1 & 2 \\
Ordnance & 6 & 2 & 3 \\
Fire control & 4 & 4 & 2 \\
Communication & 6 & 4 & 2 \\
Photoelectric & 2 & 4 & 1 \\
Electrical & 5 & 2 & 2 \\
\hline
\end{tabular}

configuration of Case 2, and use the improved FOA to solve the personnel configuration model. In order to verify the superiority of IFOA, the original FOA and genetic algorithm (GA) is used for comparative simulation with IFOA.

Set the number of iterations of all algorithms to 800 and the population size to 200. The flight direction judgment value of IFOA is $M=60$ and $M=120$. FOA does not improve; that is, all fruit fly positions and new ways only fly in the direction of global historical optimization. The crossover rate and mutation rate of the GA algorithm are set to 0.9 and 0.1 , respectively. The three algorithms of IFOA, FOA, and GA solve the case 50 times independently.

Figure 6 compares the convergence process of the three algorithms, where Figure 6(a) compares the convergence process of only one run, and Figure 6(b) compares the average convergence process of 50 runs. It can be seen that the convergence effect of IFOA algorithm is the best compared with FOA algorithm and GA algorithm. Although the convergence speed is slower than FOA algorithm, there is no obvious difference. IFOA algorithm introduces three 
TABle 5: Case 2 personnel parameters.

\begin{tabular}{|c|c|c|c|c|c|c|c|c|c|c|c|c|c|c|}
\hline \multicolumn{15}{|c|}{ Personnel } \\
\hline Group & 1 & 2 & 3 & 4 & 5 & 6 & 7 & 8 & 9 & 10 & 11 & 12 & 13 & 14 \\
\hline 1 & 0.63 & 0.60 & 0.92 & 0.70 & 0.86 & 0.85 & 0.84 & 0.66 & 0.94 & 0.83 & 0.90 & 0.88 & 0.86 & 0.60 \\
\hline 2 & 0.79 & 0.68 & 0.98 & 0.98 & 0.62 & 0.88 & 0.70 & 0.97 & 0.70 & 0.66 & 0.62 & 0.97 & 0.93 & 0.86 \\
\hline 3 & 0.62 & 0.60 & 0.79 & 0.99 & 0.68 & 0.87 & 0.95 & 0.99 & 0.67 & 0.96 & 0.94 & 0.98 & 0.83 & 0.88 \\
\hline 4 & 0.62 & 0.82 & 0.84 & 0.60 & 0.81 & 0.96 & 0.81 & 0.76 & 0.64 & 0.86 & 0.89 & 0.83 & 0.75 & 0.75 \\
\hline 5 & 0.68 & 0.94 & 0.80 & 0.69 & 0.88 & 0.99 & 0.69 & 0.92 & 0.72 & 0.83 & 0.76 & 0.95 & 0.61 & 0.97 \\
\hline 6 & 0.71 & 0.75 & 0.65 & 0.95 & 0.65 & 0.96 & 0.61 & 0.92 & 0.87 & 0.65 & 0.63 & 0.82 & 0.99 & 0.61 \\
\hline Group & 15 & 16 & 17 & 18 & 19 & 20 & 21 & 22 & 23 & 24 & 25 & 26 & 27 & 28 \\
\hline 1 & 0.95 & 0.61 & 0.77 & 0.60 & 0.99 & 0.89 & 0.65 & 0.60 & 0.72 & 0.96 & 0.66 & 0.88 & 0.94 & 0.97 \\
\hline 2 & 0.85 & 0.83 & 0.91 & 0.74 & 0.89 & 0.67 & 0.67 & 0.76 & 0.65 & 0.80 & 0.85 & 0.88 & 0.93 & 0.80 \\
\hline 3 & 0.67 & 0.69 & 0.61 & 0.73 & 0.62 & 0.91 & 0.82 & 0.74 & 0.82 & 0.98 & 0.75 & 0.84 & 0.67 & 0.66 \\
\hline 4 & 0.67 & 0.97 & 0.65 & 0.67 & 0.81 & 0.64 & 0.93 & 0.87 & 0.71 & 0.94 & 0.79 & 0.92 & 0.85 & 0.64 \\
\hline 5 & 0.82 & 0.69 & 0.72 & 0.63 & 0.81 & 0.85 & 0.96 & 0.94 & 0.80 & 0.64 & 0.88 & 0.89 & 0.88 & 0.92 \\
\hline 6 & 0.64 & 0.88 & 0.61 & 0.69 & 0.80 & 0.79 & 0.73 & 0.83 & 0.83 & 0.60 & 0.83 & 0.71 & 0.97 & 0.96 \\
\hline Group & 29 & 30 & 31 & 32 & 33 & 34 & 35 & 36 & 37 & 38 & 39 & 40 & & \\
\hline 1 & 0.67 & 0.72 & 0.74 & 0.67 & 0.77 & 0.62 & 0.61 & 0.65 & 0.85 & 0.88 & 0.76 & 0.61 & & \\
\hline 2 & 0.74 & 0.69 & 0.96 & 0.94 & 0.65 & 0.81 & 0.74 & 0.87 & 0.80 & 0.91 & 0.85 & 0.80 & & \\
\hline 3 & 0.73 & 0.97 & 0.71 & 0.68 & 0.63 & 0.63 & 0.85 & 0.79 & 0.78 & 0.71 & 0.70 & 0.97 & & \\
\hline 4 & 0.82 & 0.67 & 0.96 & 0.74 & 0.97 & 0.94 & 0.80 & 0.83 & 0.80 & 0.99 & 0.89 & 0.83 & & \\
\hline 5 & 0.80 & 0.80 & 0.77 & 0.92 & 0.60 & 0.63 & 0.61 & 0.78 & 0.66 & 0.94 & 0.75 & 0.92 & & \\
\hline 6 & 0.79 & 0.93 & 0.791 & 0.69 & 0.65 & 0.77 & 0.78 & 0.75 & 0.73 & 0.79 & 0.78 & 0.62 & & \\
\hline
\end{tabular}

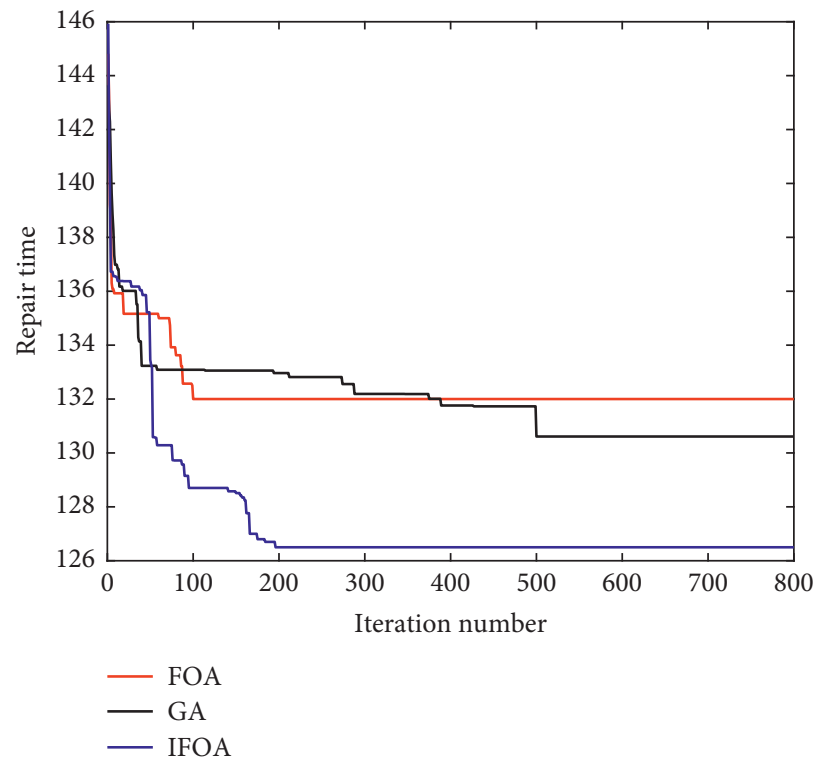

(a)

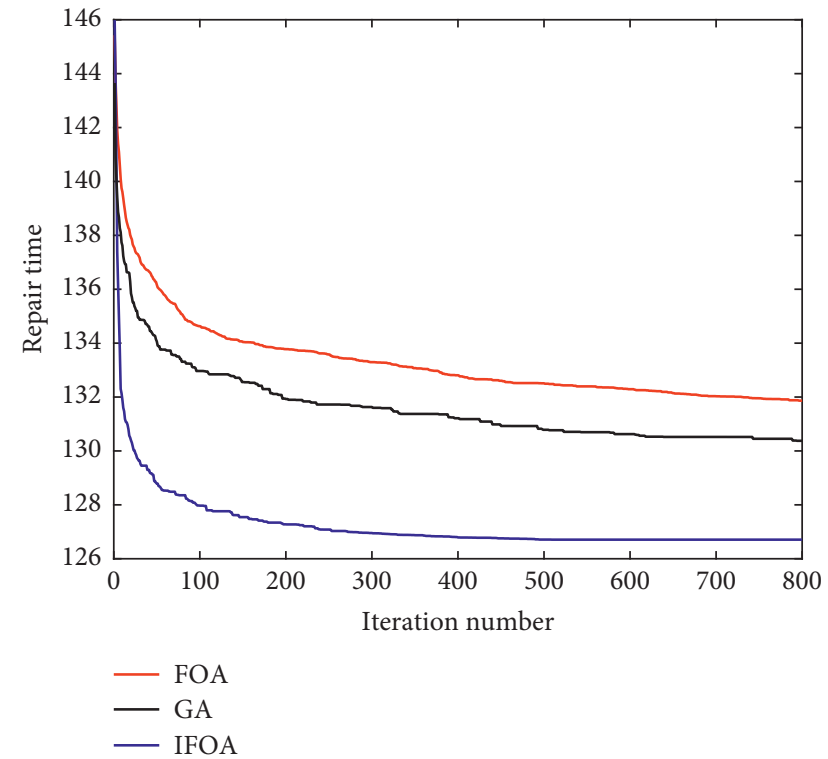

(b)

FIGURE 6: The convergence process of three algorithms. (a) One-time convergence process. (b) Average convergence process.

flight directions to improve the efficiency of the algorithm through the evolution of group collaboration. On the basis of retaining the essential optimization characteristics of the FOA, the problem that the algorithm is easy to fall into the local extreme value is solved, and the population has a good diversity, which enhances the algorithm's global optimization capability.

The maintenance personnel allocation scheme of the 22nd solution is the best solution, and the cumulative repair time of the six maintenance support teams is 126 days. Figure 7 shows the comparison of the 50-time average convergence process and the best convergence process. It can be seen that the cumulative repair time of the average convergence process is only about 0.78 days longer than the cumulative repair time of the best convergence process. The 50 -time solution result is further processed, and the result is shown in Figure 8. It can be seen that the difference between the worst solution result and the best solution result is less 


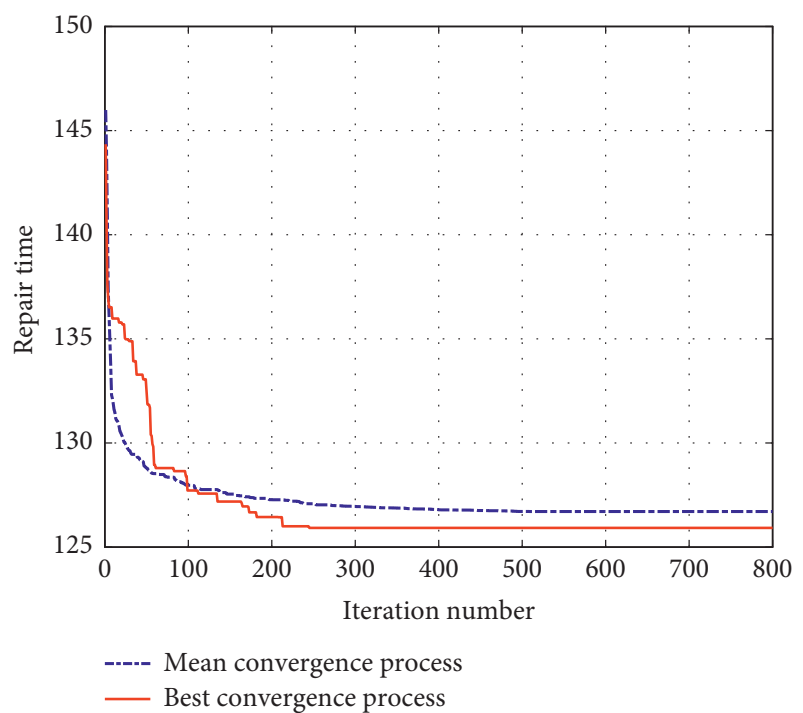

FIgURE 7: IFOA convergence process comparison.

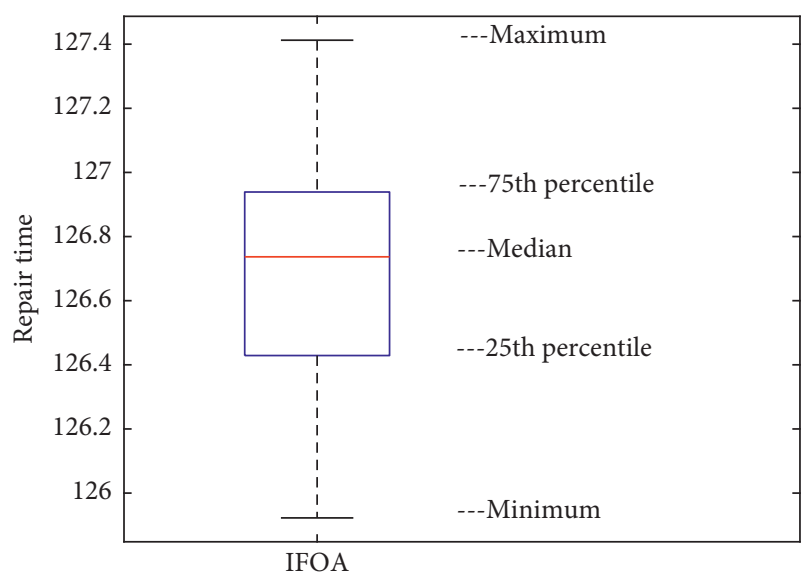

FigURE 8: IFOA convergent results' box plot.

than 1.5 days, and the interquartile range is about 0.5 days. Therefore, IFOA has strong stability and shows a good optimization effect.

Select the 22nd best solution result for specific analysis. The personnel distribution is shown in Figure 9. The maintenance personnel $9,11,15,19,20$, and 24 are assigned to group 1 to repair the vehicle equipment, responsible for repairing chassis faults. Personnel 1, 3, 12, 17, 36, 37, and 39 are assigned to work in group 2, responsible for repairing ordnance faults. Personnel 7, 8, 10, 18, 30, and 40 are assigned to work in group 3, responsible for repairing fire control faults. Personnel 16, 26, 31, 33, 34, and 38 are assigned to work in group 4, responsible for repairing communication faults. Personnel 2, 5, 6, 14, 21, 22, 25, and 32 are assigned to work in group 5, responsible for repairing photoelectric faults. The remaining personnel are assigned to work in group 6, responsible for repairing electrical faults.

According to the 22nd solution results, the maintenance personnel are assigned. The cumulative repair time of each group is shown in Table 6.

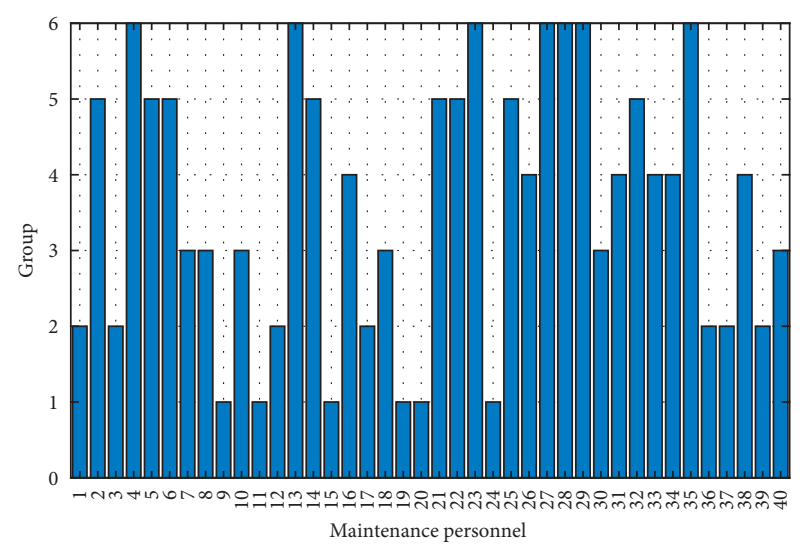

FIgURE 9: Optimal allocation scheme of maintenance personnel.

TABLE 6: Cumulative repair time of vehicle equipment.

\begin{tabular}{lcccccc}
\hline Group & 1 & 2 & 3 & 4 & 5 & 6 \\
\hline Cumulative repair time $(\mathrm{d})$ & 20 & 21 & 18 & 17 & 24 & 25 \\
\hline
\end{tabular}

\section{Conclusion}

This paper mainly studies the optimization of vehicle equipment maintenance personnel. By analyzing the relationship between maintenance support tasks and human resources, an optimization model of vehicle maintenance support personnel based on support tasks is established, using MATLAB nonlinear integer programming toolbox and improved FOA to solve two models of vehicle equipment maintenance personnel number prediction and personnel allocation optimization. In order to improve the efficiency of the traditional FOA, the group collaboration method is used to update the fruit fly position. On the basis of retaining the essential optimization characteristics of the fruit fly algorithm, the problem that the algorithm is easy to fall into the local extreme value is solved, and the global optimization capability of the algorithm is improved. The simulation results show that the improved FOA has good solution efficiency, and the personnel allocation scheme effectively shortens the repair time of the equipment. The method proposed in this paper can avoid the waste of human resources, maximize maintenance efficiency, and provide a theoretical basis for the optimal decision-making of maintenance personnel.

\section{Data Availability}

The data used to support the findings of this study are included within the article.

\section{Conflicts of Interest}

The authors declare that they have no conflicts of interest.

\section{References}

[1] J. Wang, W. S. Li, L. C. Li, and P. Wang, "Research on equipment support force prensent situation and 
countermeasure," Applied Mechanics and Materials, vol. 644650, pp. 5817-5820, 2014.

[2] C. Zhang, "Modeling and optimization of personnel allocation for equipment system maintenance and support," in Proceedings of the 2018 2nd International Conference on Management Engineering, Software Engineering and Service Sciences, pp. 73-76, Wuhan, China, January 2018.

[3] F. Kelvin, "Infantry fighting vehicle-protecting your troops for combat and peacekeeping duties," Asian Defence Journal, pp. 6-10, 2016.

[4] E. B. Tirkolaee, A. Goli, M. Hematian, A. K. Sangaiah, and T. Han, "Multi-objective multi-mode resource constrained project scheduling problem using Pareto-based algorithms," Computing, vol. 101, no. 6, pp. 547-570, 2019.

[5] S. C. Solving, "The stochastic multimode resource-constrained project scheduling problem," in Proceedings of the PMS 2020: 17th International Conference on Project Management and Scheduling, Jaipur, Rajasthan, India, April 2020.

[6] M. Zhiqiang, H. Zhengwen, W. Nengmin et al., "A genetic algorithm for the proactive resource-constrained project scheduling problem with activity splitting," IEEE Transactions on Engineering Management, vol. 66, pp. 459-474, 2019.

[7] S. Creemers, "The preemptive stochastic resource-constrained project scheduling problem," European Journal of Operational Research, vol. 277, no. 1, 2019.

[8] A. Delgoshaei, S. Esmaeili Hanjani, and A. Hossein Nasiri, "A genetic algorithm for scheduling multimode resource-constrained project problem in the presence of preemptive resources," Journal of Project Management, vol. 4, no. 3, pp. 195-212, 2019.

[9] Y. Jin, H. Nie, P. Duan et al., "An Improved Cuckoo search algorithm for resource-constrained project scheduling problem with generalized precedence relations," in Proceedings of the Intelligent Control \& Automation, Guilin, China, June 2016.

[10] H. Dai, W. Cheng, and P. Guo, “An improved Tabu search for multi-skill resource-constrained project scheduling problems under step-deterioration," Arabian Journal for Science \& Engineering, vol. 43, pp. 3279-3290, 2018.

[11] H. Q. Zhang, Y. C. Lu, and M. Wang, "Optimal allocation of crew support in war time ships based on hybrid genetic algorithm," Journal of Gun Launch and Control, vol. 44, no. 4, pp. 37-43, 2019.

[12] J. Xia, Y. Feng, X. Xue et al., "Multi-echelon inventory allocation of multi-indenture spare parts considering maintenance ratio," IOP Conference Series Materials Science and Engineering, vol. 715, Article ID 012047, 2020.

[13] Z. Shuai, T. Ke-Nan, L. Wei-Min et al., "Multi-echelon and multi-indenture inventory optimal model for carrier-based aircraft repairable spare parts based on mission and task," Science Technology and Engineering, vol. 15, no. 2, pp. 298303, 2015.

[14] L. Zhou, Y. Peng, Q. Li et al., "Research on the general evaluation model of task oriented spare parts supply," Xi Tong Gong Cheng Yu Dian Zi Ji Shu/Systems Engineering \& Electronics, vol. 39, no. 9, pp. 2046-2051, 2017.

[15] J. Li and Z. H. Ji, "Joint replenishment problem of military aircraft consumptive items under resource constraint," in Proceedings of the 2017 8th International Conference on Mechanical and Aerospace Engineering (ICMAE), pp. 786790, Prague, Czech Republic, July 2017.

[16] C. Jing, Y. Yibing, Z. Li et al., "Joint optimization of preventive maintenance and spare parts inventory with appointment policy," Mathematical Problems in Engineering, vol. 2017, Article ID 3493687, 12 pages, 2017.

[17] T. Yan, Y. Lei, B. Wang et al., "Joint maintenance and spare parts inventory optimization for multi-unit systems considering imperfect maintenance actions," Reliability Engineering and System Safety, vol. 202, 2020.

[18] X. Wang, "Research on the division of internal responsibilities and the rationalization of personnel allocation in financial departments," Finance and Market, vol. 5, no. 4, p. 276, 2020.

[19] F. Santos, R. Fukasawa, and L. Ricardez-Sandoval, "An integrated machine scheduling and personnel allocation problem for large-scale industrial facilities using a rolling horizon framework," Optimization and Engineering, no. 4, 2020.

[20] H. B. Yin, A. M. Zhang, and L. Gong, "Demand calculation of wartime vehicle equipment maintenance power based on minimum maintenance unit," Journal of Military Transportation University, vol. 18, no. 2, pp. 29-32, 2016.

[21] M. Y. Luo and T. Liu, "Research on the model of equipment maintenance personnel demand in the troops," Journal of Academy of Equipment, vol. 24, no. 2, pp. 44-47, 2013.

[22] S.-W. Kim and B.-K. Yoon, "A study on the optimal allocation of maintenance personnel in the naval ship maintenance system," Journal of the Korea Academia-Industrial Cooperation Society, vol. 16, no. 3, pp. 1853-1862, 2015.

[23] L. H. Shi, J. Chen, Z. Q. Ye et al., "Requirement analysis of POL equipment maintenance personnel based on queuing theory," Command Control \& Simulation, vol. 41, no. 1, pp. 136-140, 2019.

[24] X. Su, W. Han, Y. Wu et al., "A proactive robust scheduling method for aircraft carrier flight deck operations with stochastic durations," Complexity, vol. 2018, Article ID 6932985, 38 pages, 2018.

[25] L. Yue, "Equipment support resources forecasting method research based on the uncertainty of equipment operation activities' time sequence," in Proceedings of the 2014 Prognostics and System Health Management Conference (PHM2014 Hunan), pp. 607-611, Hunan, China, August 2014.

[26] Y. B. Gu and Y. X. Jia, "A study on maintenance task based maintenance manpower prediction model," Fire Control and Command Control, vol. 38, no. 11, pp. 107-110, 2013.

[27] T. H. Wu, C. L. Chen, P. Ding et al., "Method of determining quantity of wartime maintenance support personnel," Journal of Academy of Armored Force Engineering, vol. 32, no. 5, pp. 33-36, 2018.

[28] Y. Wang, "Predication and optimization of maintenance resources for weapon system," International Journal of Intelligent Systems and Applications, vol. 3, no. 5, 2011.

[29] S. H. Sheu, S. H. Li, and C. C. Chang, "A generalised maintenance policy with age-dependent minimal repair cost for a system subject to shocks under periodic overhaul," International Journal of Systems Science, vol. 43, no. 6, pp. 1007-1013, 2012.

[30] G. Tianshan and G. Bo, "Failure rate prediction of substation equipment combined with grey linear regression combination model," in Proceedings of the IEEE International Conference on High Voltage Engineering \& Application, pp. 1-5, Chengdu, China, September 2016.

[31] J. Wang and H. Yin, "Failure rate prediction model of substation equipment based on Weibull distribution and time series analysis," IEEE Access, vol. 7, no. 99, p. 1, 2019.

[32] J. S. Zhao, C. Y. Linghu, and Y. He, "Repair workload measuring model of wartime vehicle equipment," Journal of 
Military Transportation Institute, vol. 18, no. 6, pp. 27-31, 2016.

[33] J. Cui, Y. Wu, X. Su, and J. Song, A task allocation model for a team of aircraft launching on the carrier," Mathematical Problems in Engineering, vol. 2018, Article ID 7920806, 12 pages, 2018.

[34] Y. Wu, "A survey on population-based meta-heuristic algorithms for motion planning of aircraft," Swarm and Evolutionary Computation, vol. 62, no. 6, Article ID 100844, 2021.

[35] J. C. Bansal, P. K. Singh, and N. R. Pal, Evolutionary and Swarm Intelligence Algorithms, Springer, Berlin, Germany, 2019.

[36] D. Shan, G. Cao, and H. Dong, "LGMS-FOA: an improved fruit fly optimization algorithm for solving optimization problems," Mathematical Problems in Engineering, vol. 2013, Article ID 108768, 9 pages, 2013. 